\title{
A pandemia da Covid-19 e o adoecimento mental da gestante
}

The Covid-19 pandemic and the mental illness of pregnant womem

La pandemia de Covid-19 y la enfermedad mental de las mujeres embarazadas

Samara Cristina Lima Sousa

ORCID: https://orcid.org/0000-0003-3224-6569 Faculdade Estácio de Teresina, Brasil E-mail: samaracristina129@gmail.com

Alice Pereira Dias

ORCID: https://orcid.org/0000-0001-8048-345X Faculdade Estácio de Teresina, Brasil

E-mail: alicedias1@outlook.com.br

Kayron Rodrigo Ferreira Cunha

ORCID: https://orcid.org/0000-0003-3507-3376 Universidade Federal do Piauí, Brasil E-mail: ikayron.kr@gmail.com

Kelven Rubson Soares Bezerra

ORCID: https://orcid.org/0000-0002-0680-9295

Faculdade Evangélica do Meio do Norte, Brasil E-mail: kelvenrub5@gmail.com

Amanda de Oliveira Lima

ORCID: https://orcid.org/0000-0002-0955-5542 Universidade Estadual do Piauí, Brasil

E-mail: amandaolimapsi@gmail.com

Silvania Maria Cunha do Nascimento

ORCID: https://orcid.org/0000-0002-1940-4588

Faculdade de Ciências e Tecnologia, Brasil

E-mail: silvaniamaria.cunha@gmail.com

Maycon Guimarães Santos

ORCID: https://orcid.org/0000-0003-1089-0417

Universidade Estadual do Piauí, Brasil

E-mail: ma1987ycon@hotmail.com

Jucelene Soares de Macedo

ORCID: https://orcid.org/0000-0002-6537-7410 Faculdade Futura, Brasil

E-mail: jsmmacedo@gmail.com

Francisco Robson de Oliveira Alves

ORCID: https://orcid.org/0000-0002-9854-6460

Universidade Federal do Piauí, Brasil

E-mail: robson_oliveira.fisio@outlook.com

Kyvia Naysis de Araujo Santos

ORCID: https://orcid.org/0000-0003-3707-986X

Universidade Federal do Piaú, Brasil

E-mail: kyvianaysis@gmail.com

Amanda Fernanda Damasceno Saraiva de Sousa

ORCID: https://orcid.org/0000-0003-2395-8353

Universidade Federal do Piauí, Brasil

E-mail: amandamascpsi@gmail.com

Nanielle Silva Barbosa

ORCID: https://orcid.org/0000-0001-5758-2011

Universidade Estadual do Piaú, Brasil

E-mail: naniellesilvabarbosa@hotmail.com

\section{Resumo}

Este estudo traz como objetivo discutir, com base na literatura científica, o adoecimento mental das gestantes durante a presente pandemia da Covid-19. Trata-se de um estudo de revisão integrativa, com busca realizada na Base de Dados de Enfermagem, Literatura Latino-americana e do Caribe em Ciências da Saúde, Índice Bibliográfico Español en Ciencias de la Salud, Medical Literature on Line e Cumulative Index to Nursing and Allied Health Literature, durante os meses de outubro e novembro de 2020. Utilizou-se os descritores: "Gestantes", "Transtornos mentais" e 'Coronavírus', Após triagem das produções, cinco foram incluídas como amostra do estudo. Das publicações incluídas, todas eram de abordagem quantitativa. Três publicações foram classificadas com Nível de Evidência III 
(ensaio clínico bem delineado sem randomização). Trouxeram como principais resultados a presença de sintomas de ansiedade, depressão e estresse e seus impactos negativos que contribuem para o sofrimento psíquico da gestante durante o período pandêmico. Durante a pandemia da Covid-19, a gestante encontra-se em maior vulnerabilidade e pode sofrer com o adoecimento mental, com destaque para o aparecimento de sintomas relacionados a transtornos mentais.

Palavras-chave: Infecções por Coronavírus; Gravidez; Saúde mental; Transtornos mentais.

\begin{abstract}
This study aims to discuss, based on scientific literature, the mental illness of pregnant women during the current Covid-19 pandemic. This is an integrative review study, with a search carried out in the Nursing Database, Latin American and Caribbean Literature on Health Sciences, Bibliographic Index Español en Sciences de la Salud, Medical Literature on Line and Cumulative Index to Nursing and Allied Health Literature, during the months of October and November 2020. The following descriptors were used: "Pregnant women", 'Mental disorders' and "Coronavirus". After screening the productions, five were included as a study sample. Of the publications included, all had a quantitative approach. Three publications were classified as Level of Evidence III (well-designed clinical trial without randomization). The main results were the presence of symptoms of anxiety, depression and stress and their negative impacts that contribute to the psychological distress of pregnant women during the pandemic period. During the Covid-19 pandemic, pregnant women are more vulnerable and may suffer from mental illness, with emphasis on the appearance of symptoms related to mental disorders.
\end{abstract}

Keywords: Coronavirus infections; Pregnancy; Mental health; Mental disorders.

\title{
Resumen
}

Este estudio tiene como objetivo discutir, basado en la literatura científica, la enfermedad mental de las mujeres embarazadas durante la actual pandemia de Covid-19. Se trata de un estudio de revisión integradora, con búsqueda realizada en la Base de Datos de Enfermería, Literatura Latinoamericana y del Caribe en Ciencias de la Salud, Índice Bibliográfico Español en Ciencias de la Salud, Literatura Médica en Línea e Índice Acumulativo de Literatura de Enfermería y Afines en Salud., durante los meses de octubre y noviembre de 2020. Se utilizaron los siguientes descriptores: " Mujeres embarazadas ", 'Trastornos mentales' y " Coronavirus ". Tras la proyección de las producciones, se incluyeron cinco como muestra de estudio. De las publicaciones incluidas, todas tuvieron un enfoque cuantitativo. Tres publicaciones se clasificaron como Nivel de evidencia III (ensayo clínico bien diseñado sin aleatorización). Los principales resultados fueron la presencia de síntomas de ansiedad, depresión y estrés y sus impactos negativos que contribuyen al malestar psicológico de las embarazadas durante el período pandémico. Durante la pandemia de Covid-19, las mujeres embarazadas son más vulnerables y pueden padecer enfermedades mentales, con énfasis en la aparición de síntomas relacionados con los trastornos mentales.

Palabras clave: Infecciones por Coronavirus; Embarazo; Salud mental; Trastornos mentales.

\section{Introdução}

A doença do novo coronavírus (Sars-CoV-2), denominada Covid-19, transcorreu com a identificação dos primeiros casos de infectados em dezembro de 2019, em Wuhan, localizada na província de Hubei, China. No Brasil, o relato dos primeiros casos ocorreu em fevereiro de 2020. A partir de então ações passaram a ser implementadas com o objetivo de conter a rápida disseminação do vírus. Coronavírus apresentam ácido ribonucleico (RNA) envelopado, geralmente encontrados em humanos, mamíferos e aves, capazes de causar diversos tipos de doenças, mais especificamente doenças respiratórias graves (Oliveira et al., 2020; Lana et al., 2020).

A disseminação da doença surpreendeu pela ampla velocidade com a qual alcançou os diversos países do globo terrestre. O número de pessoas infectadas em um curto período de tempo e os óbitos provocados impressionam. Há grandes preocupações acerca da duração imprecisa deste episódio, o que traz significativas mudanças para o convívio social, com transformações a nível global nos hábitos de vida e maior sensibilização para seu controle (Souza, 2020).

Com a chegada dessa pandemia, passou-se a ter uma atenção especial e redobrada aos grupos considerados de risco, pela presença de fatores que possibilitam o desenvolvimento de formas mais graves da doença. Conforme classificação da Organização Mundial da Saúde (OMS) mulheres grávidas encontram-se como um desses principais grupos, destacando a maior vulnerabilidade das gestantes de alto risco com possibilidade de manifestar pior evolução da infecção em razão de comorbidades pré-existentes (Brasil, 2020). 
Na gravidez, as mulheres tornam-se mais suscetíveis à infecções por patógenos respiratórios e pneumonias graves. Isso se deve às alterações imunológicas e modificações fisiológicas para se adaptar a gestação. Além dessas alterações, o período gravídico-puerperal também é favorável ao sofrimento mental e ao desenvolvimento de transtornos que podem afetar a saúde da mulher. O diagnóstico nesta etapa é complexo, havendo poucos estudos que elucidam o tema no decorrer da gravidez e suas repercussões para a mãe e o feto (Furlan et al., 2020; Almeida, Portugal \& Assis, 2020).

A vulnerabilidade ocasionada pelas mudanças gestacionais predispõe um elevado número de mulheres ao desenvolvimento de diversas desordens psicológicas, sendo muito comum o aparecimento de um quadro de depressão com episódios depressivos, acarretando prejuízos relacionado ao bem-estar fetal, interferências no tratamento farmacológico e prejuízo de atividades básicas da vida diária da gestante. Outros tipos de transtornos podem ser manifestados neste período, incluindo ansiedade, mudanças de humor e do pensamento ou sintomas de origem somática (Souza et al., 2017).

Todavia, por ser um problema de saúde pública recente, estudos relacionados ao sofrimento mental vivenciado por grávidas durante a pandemia do Sars-Cov-2 ainda são escassos e limitados, dificultando a adoção de medidas e intervenções assistenciais baseadas em evidências. Assim considerando, este estudo traz como objetivo discutir, com base na literatura científica, o adoecimento mental das gestantes durante a presente pandemia da Covid-19.

\section{Metodologia}

Trata-se de uma revisão integrativa da literatura com construção baseada em seis etapas (Mendes, Silveira \& Galvão, 2008). Definiu-se como questão norteadora, por meio da estratégia PICo (População: gestantes; Interesse: adoecimento mental; Contexto: pandemia da Covid-19): "o que trazem as evidências científicas acerca do adoecimento mental de gestantes durante a pandemia da Covid-19?'” (Loockwood et al., 2017).

Realizou-se a busca na literatura entre outubro e novembro de 2020 na Base de Dados de Enfermagem (BDENF), Literatura Latino-americana e do Caribe em Ciências da Saúde (LILACS), Índice Bibliográfico Español en Ciencias de la Salud (IBECS), Medical Literature on Line (MEDLINE) e Cumulative Index to Nursing and Allied Health Literature (CINAHL), utilizando-se os Descritores em Ciências da Saúde (DeCS) e seus correspondentes no Medical Subject Headings (MeSH) e list CINAHL: "Gestantes", "Transtornos mentais" e "Coronavírus" combinados com o operador boleano AND.

Instituíram-se como critérios de inclusão: estudos primários em português, inglês e espanhol, publicados no ano de 2020, tal delimitação cronológica está relacionada ao período decorrente da pandemia ocasionada pelo novo coronavírus. Foram excluídos teses, dissertações, revisões, editoriais e artigos de opinião. A seleção foi desenvolvida por dois revisores, independentemente, em duas etapas.

Na primeira etapa, realizou-se a leitura de título e resumo das produções a fim de identificar se estavam diretamente relacionadas ao objetivo proposto e, na segunda etapa, fez-se a leitura do texto completo a fim de extrair e analisar informações importantes para a construção da síntese dos resultados e discussão. Nos casos em que houve discordância, a opinião de um terceiro revisor foi solicitada.

Para a extração dessas informações, consideradas fundamentais, um instrumento de coleta de dados foi elaborado pelas autoras em uma planilha no programa Microsoft Office Word ${ }^{\circledR}$, contendo as seguintes variáveis: título, autoria, abordagem metodológica, principais resultados encontrados na pesquisa e nível de evidência (NE) (Whittemore \& Knafl, 2005).

A fim de melhor compreensão, o percurso de elegibilidade de cada estudo que compôs a amostra final dessa revisão é demonstrado no fluxograma embasado no Preferred reporting items for systematic reviews and meta-analyses (PRISMA), representado na Figura 1: 
Figura 1: Fluxograma do processo de identificação e seleção de artigos.

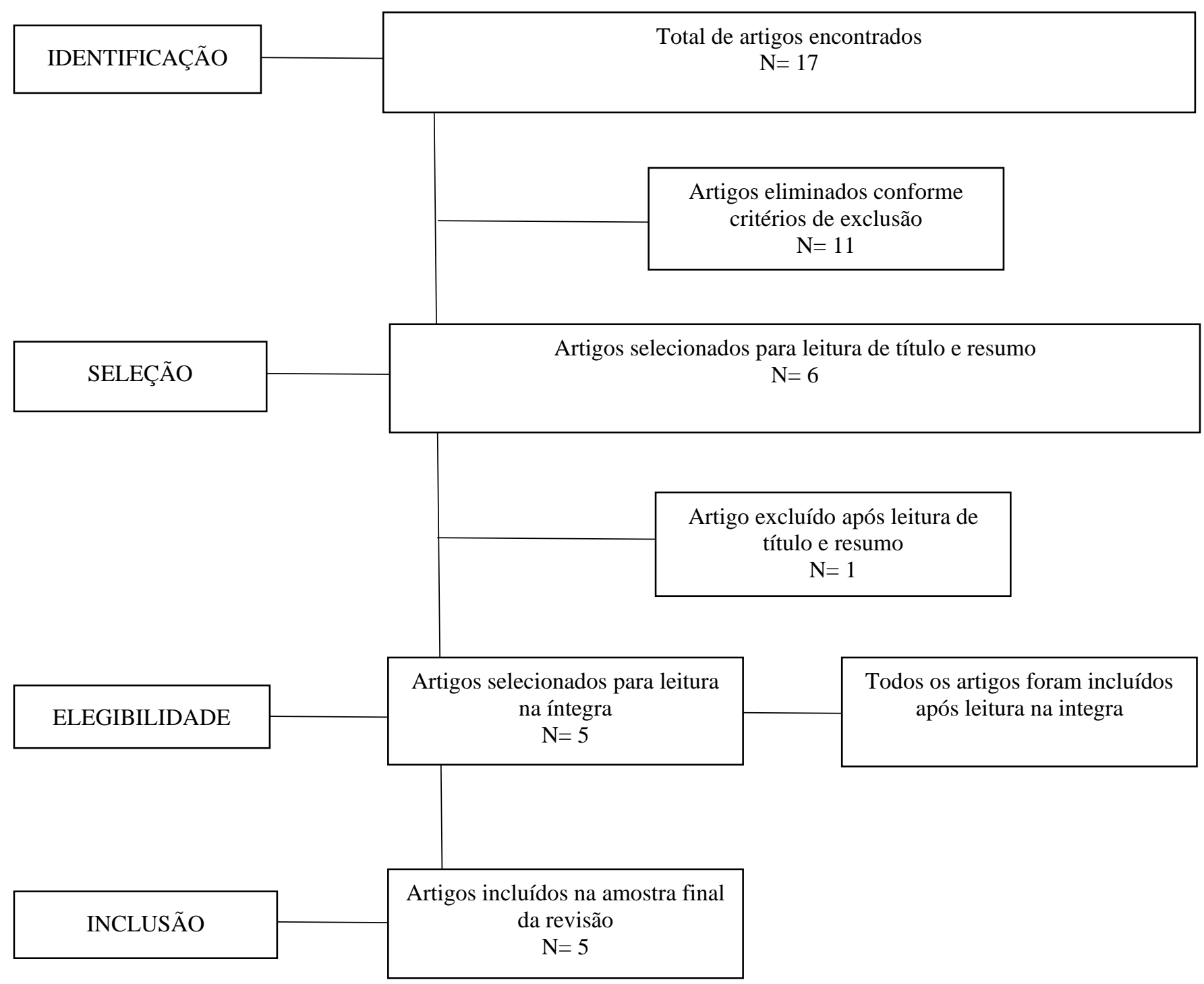

Fonte: Elaborado pelo autor a partir do Fluxograma PRISMA (2021).

\section{Resultados e Discussão}

Das publicações incluídas, todas eram de abordagem quantitativa. Três publicações foram classificadas com NE III (ensaio clínico bem delineado sem randomização). Trouxeram como principais resultados a presença e impactos de sinais e sintomas relacionados a sofrimento psíquico em gestantes durante a pandemia da Covid-19, conforme Tabela 1. 
Tabela 1: Síntese dos estudos incluídos, categorizados por título, autoria, abordagem metodológica, NE e principais resultados.

\begin{tabular}{|c|c|c|c|}
\hline Título & Autoria & Abordagem metodológica/NE & Principais resultados \\
\hline $\begin{array}{l}\text { Uptrend in distress and } \\
\text { psychiatric } \\
\text { symptomatology in } \\
\text { pregnant women during } \\
\text { the coronavirus disease } \\
2019 \text { pandemic }\end{array}$ & Berthelot et al. & $\begin{array}{l}\text { Dois coortes de mulheres grávidas } \\
(\mathrm{n}=1.754) \text {, recrutadas em clínicas de } \\
\text { pré-natal, antes e após inicío da } \\
\text { pandemia, em Quebec, Canadá. } \mathrm{NE}=\mathrm{IV}\end{array}$ & $\begin{array}{l}\text { Mulheres grávidas avaliadas durante } \\
\text { a pandemia COVID-19 relataram } \\
\text { maior sofrimento e sintomas } \\
\text { psiquiátricos, com destaque para } \\
\text { ansiedade e depressão. }\end{array}$ \\
\hline $\begin{array}{l}\text { The prevalence of } \\
\text { psychiatric symptoms of } \\
\text { pregnant and non- } \\
\text { pregnant women during } \\
\text { the COVID-19 epidemic }\end{array}$ & Zhou et al. & $\begin{array}{l}\text { Estudo transversal realizado com } \\
\text { mulheres grávidas }(\mathrm{n}=544) \text { e não } \\
\text { grávidas }(\mathrm{n}=315) \text { em hospitais materno- } \\
\text { infantil de Pequim, China, entre } \\
\text { Fevereiro a Março de 2020. NE=III }\end{array}$ & $\begin{array}{l}\text { Durante a epidemia de COVID-19 na } \\
\text { China, as mulheres grávidas } \\
\text { apresentaram melhor enfrentamento } \\
\text { em relação a problemas mentais, } \\
\text { demostrando menores índices de } \\
\text { depressão, ansiedade, e insônia. }\end{array}$ \\
\hline $\begin{array}{l}\text { A Mixed-Methods Pilot } \\
\text { Study of Perinatal } \\
\text { Risk and Resilience } \\
\text { During COVID-19 }\end{array}$ & Farewe & $\begin{array}{l}\text { Estudo exploratório online com } 37 \\
\text { mulheres (gestantes e puérperas), entre } \\
\text { Março à Abril de } 2020 \text {, no Colorado, } \\
\text { Estados Unidos da América. NE=VI }\end{array}$ & $\begin{array}{l}\text { As entrevistadas relataram que desde } \\
\text { o início da pandemia da COVID-19, } \\
\text { Apresentaram sintomatologia de } \\
\text { estresse, depressão e ansiedade. }\end{array}$ \\
\hline $\begin{array}{l}\text { Elevated depression and } \\
\text { anxiety symptoms } \\
\text { among pregnant } \\
\text { individuals during the } \\
\text { COVID-19 pandemic }\end{array}$ & Lebel et al. & $\begin{array}{l}\text { Estudo desenvolvido online com } 1987 \\
\text { mulheres grávidas em Abril de } 2020 \text { no } \\
\text { Canadá. NE=III }\end{array}$ & $\begin{array}{l}\text { Sintomas elevados de ansiedade e } \\
\text { depressão foram identificados entre } \\
\text { as grávidas durante a pandemia de } \\
\text { COVID-19. }\end{array}$ \\
\hline $\begin{array}{l}\text { Risk for Depressive } \\
\text { Symptoms among } \\
\text { Hospitalized Women in } \\
\text { High-Risk Pregnancy } \\
\text { Units during the } \\
\text { COVID-19 Pandemic }\end{array}$ & Sade et al. & $\begin{array}{l}\text { Estudo transversal comparativo entre } \\
\text { gestantes de alto risco hospitalizadas no } \\
\text { Soroka University Medical } \\
\text { Center,durante a pandemia ( } \mathrm{n}=90) \text { e } \\
\text { anteriormente à ela }(\mathrm{n}=279) \text {. NE=III }\end{array}$ & $\begin{array}{l}\text { As mulheres gestantes hospitalizadas } \\
\text { durante a pandemia da COVID-19 } \\
\text { não apresentaram risco aumentado de } \\
\text { depressão em comparação com as } \\
\text { hospitalizadas antes da pandemia. }\end{array}$ \\
\hline
\end{tabular}

Fonte: Autores (2021).

Com os estudos obtidos por meio da busca sistematizada na literatura, observa-se que grande parte das gestantes apresentaram algum tipo de fragilidade psicológica, agravada em virtude da pandemia da Covid-19. Esses aspectos favoreceram o desenvolvimento de transtornos psicológicos que podem interferir no curso natural da gestação e afetar negativamente as relações mãe, bebê e família.

A mulher, no decorrer da fase gestacional, está exposta a uma série de alterações, tanto físicas quanto psíquicas e sociais. Dentre essas, destaca-se a maior fragilidade de sua saúde mental. Portanto, é considerado um período propício ao aparecimento ou agravamento de sintomatologias relacionadas a sofrimento mental e, mais gravemente, o surgimento de transtornos mentais, especialmente no primeiro e terceiro trimestres da gestação e nos primeiros 30 dias do puerpério (Guimarães et al., 2019).

Estudos apontam quem com a disseminação do SARS-CoV-2 pelo mundo, as mulheres grávidas foram diretamente afetadas pelo estresse e ansiedade, principalmente pela preocupação excessiva em relação ao risco de exposição e prejuízos ao feto. Esses transtornos associam-se a impactos negativos no curso fisiológico da gravidez, como pré-eclâmpsia, depressão, aumento de náuseas e vômitos, trabalho de parto prematuro, baixo Apgar e peso ao nascer do bebê (Berthelot et al., 2020; Zhou et al., 2020; Farewell et al., 2020; Lebel et al., 2020; Furlan et al., 2020; Costa et al., 2018).

Estudo exploratório realizado com 37 mulheres grávidas, no Colorado, Estados Unidos da América (EUA) evidenciou que sentimentos como abandono e solidão estiveram associado ao isolamento social. Tal "confinamento" é fator primordial para a ocorrência de ansiedade, depressão, uso abusivo de psicotrópicos e menor bem-estar mental. Além dos problemas que podem vim a acontecer, estudos acerca da transmissão vertical ainda são excassos e inconclusivos, consequentemente, mulheres ficam receosas, sendo prejudicial à sua saúde mental (Farewell et al., 2020; Lélis et al., 2020). 
Ainda em relação ao isolamento social, a permissão de acompanhantes durante o parto também foi prejudicada, o que gera maior sofrimento para a mulher, considerando que, no momento do nascimento, a parturiente necessita de apoio emocional e se sentir acolhida e segura, principalmente se esta for diagnosticada com o novo coronavírus. Enfatiza-se o papel crucial da equipe de saúde na prestação de um cuidado integral e humanizado (Estrela et al., 2020).

Entretanto, algumas evidências trouxeram um panorama oposto ao contextualizado até o momento. Uma investigação entre mulheres grávidas e não grávidas durante a pandemia, apontou que as gestantes tiveram menores chances para desenvolver sintomatologias psiquiátricas. Aspectos relacionados à condição socioeconômica e psicológica antes de engravidar, redes de apoio familiar bem estruturadas, acesso à assistência de qualidade e alterações nas cargas hormonais, podem estar associadas a esse desfecho (Zhou et al., 2020).

Outra investigação, a fim de identificar sintomas depressivos em gestantes hospitalizadas, em unidades de alto risco durante a pandemia da Covid-19, demonstrou que não houve diferença expressiva de casos de depressão, fato que pode ser associado a capacidade dessas mulheres no enfrentamento a situação difíceis, mesmo em casos de desastres naturais, a estimulação do desenvolvimento de valorização da vida, melhorias nas relações interpessoais e autossuperação após tragédias (Sade et al., 2020).

Todavia, ressalta-se a importância da atenção e cuidado especial a esse grupo de risco, visto que além da fragilidade identificada no período gravídico-puerperal, encontra-se ainda todas as aflições e angústias ocasionadas pelo atual cenário de saúde. Nesse contexto, é necessário a implantação de intervenções que possam amenizar os impactos da Covid-19 na saúde da gestante e do feto, com a finalidade de possibilitar qualidade de vida e bem-estar a mulher durante esta fase da vida (Estrela et al., 2020).

Mediante a essa pandemia, os profissionais de saúde devem assegurar à gestante uma assistência de qualidade, do prénatal ao puerpério, bem como à criança, garantindo seus direitos para um nascimento seguro e crescimento e desenvolvimento saudáveis. Recomenda-se então, o estímulo às medidas de prevenção, avaliação e classificação dos riscos, orientações e educação em saúde, bem como o acompanhamento e realização de um pré-natal adequado e satisfatório que vise a saúde biopsicossocial da mulher (Mascarenhas et al., 2020).

O acompanhamento pré-natal foi apontado como um diferencial em relação às disfunções psiquiátricas no contexto da pandemia da Covid-19, onde percebeu-se que, em gestantes que tiveram acesso restrito as consultas, sintomas de ansiedade estiveram mais exacerbados (Lebel et al., 2020).

Em síntese, uma rede de apoio fortalecida é fundamental para o enfrentamento do sofrimento mental e promoção do bem-estar psíquico e social durante a gravidez, uma vez que a mulher pode necessitar de suporte, orientações e apoio emocional. Apesar das recomendações de distanciamento e isolamento, a internet e as redes sociais podem ser utilizadas como ferramentas facilitadoras na aproximação e no estabelecimento de relações benéficas, porém, tendo-se atenção e cuidado quanto ao acesso e disseminação de Fake News, que podem influenciar negativamente na manutenção da saúde mental (Almeida, Portugal \& Assis, 2020; Galhardi et al., 2020).

Como limitações para o desenvolvimento deste estudo, destaca-se a escassez de produções científicas relacionadas ao sofrimento mental em gestantes durante a pandemia da Covid-19, com atenção para a carência de produções à nível nacional e internacional, dificultando, assim, uma abordagem e entendimento mais aprofundado sobre o tema. Logo, esta produção visa contribuir com o meio científico a medida que apresenta lacunas no conhecimento a serem superadas, buscando o cuidado integral e holístico a mulher e ao seu bebê em todas as fases do processo do gestar e parir. 


\section{Considerações Finais}

Durante a pandemia da Covid-19 a gestante encontra-se em maior vulnerabilidade e pode sofrer com o adoecimento mental, com destaque para o aparecimento de sintomas relacionados a transtornos mentais como estresse, ansiedade e depressão. Esse sofrimento pode comprometer sua saúde, bem-estar e qualidade de vida, bem como como sua relação com o feto, na construção do vínculo materno-fetal e, com as redes sociais que a cercam.

Nesse contexto, as mulheres grávidas necessitam de uma assistência eficiente e eficaz, garantindo um cuidado integral, humanizado e holístico, com o intuito de implementar intervenções que possam acolhê-las e escutá-las, identificando suas reais necessidades e fatores de risco para o surgimento de transtornos mentais, garantindo assim um período gravídicopuerperal saudável.

A partir desta reflexão, faz-se necessário a criação de políticas públicas que visem solucionar este problema de saúde pública, juntamente com a ampliação de ações conjuntas trabalhadas mais especificamente durante o pré-natal voltadas para o fortalecimento das redes de apoio, como rodas de conversas e oficinas terapêuticas, possibilitando identificar e avaliar gestantes com maior vulnerabilidade psicológica, social e econômica. Essa estratificação é extremamente necessária pois, auxilia o direcionamento a ser seguido, contribuindo para um pré-natal de qualidade.

\section{Referências}

Almeida, M. O., Portugal, T. M. \& Assis, T. J. C. F. (2020). Gestantes e COVID-19: isolamento como fator de impacto físico e psíquico. Rev. Bras. Saúde Mater. Infant, 20(2): 603-606.

Berthelot, N., Lemieux, R., Garon-Bissonnette, J., Drouin-Maziade, C., Martel, E. \& Maziade, M. (2020). Uptrend in distress and psychiatric symptomatology in pregnant women during the coronavirus disease 2019 pandemic. Acta Obstet Gynecol Scand, 99: 848-855.

Brasil. Ministério da Saúde. (2020). Secretaria de Atenção Primária à Saúde. Departamento de Ações Programáticas e Estratégicas. Nota Informativa n 13/2020 - SE/GAB/SE/MS. Manual de Recomendações para a Assistência à Gestante e Puérpera frente à Pandemia de Covid-19.

Costa, D.O., Souza, F. I. S., Pedroso, G. C. \& Strufaldi, M. W. L. (2018). Transtornos mentais na gravidez e condições do recém-nascido: estudo longitudinal com gestantes assistidas na atenção básica. Ciência \& Saúde Coletiva, 23(3): 691-700.

Estrela, F. M., Silva, K. K. A., Cruz, M. A. \& Gomes, N. P. (2020). Gestantes no contexto da pandemia da Covid-19: reflexões e desafios. Physis, 30(2): e300215. http://dx.doi.org/10.1590/S0103-73312020300215

Farewell, C. V., Jewell, J., Walls, J. \& Leiferman, J. A. (2020). A Mixed-Methods Pilot Study of Perinatal Risk and Resilience During COVID-19. J Prim Care Community Health, 11: 2150132720944074.

Furlan, M. C. R., Jurado, S. R., Uliana, C. H., Silva, M. E. P., Nagata, L. A. \& Maia, A. C. F. (2020). Gravidez e infecção por coronavírus: desfechos maternos, fetais e neonatais - Revisão sistemática. Revista Cuidarte, 11(2): e1211.

Galhardi, C. P., Freire, N. P., Minayo, M. C. S. \& Fagundes, M. C. M. (2020). Fato ou Fake? Uma análise da desinformação frente à pandemia da Covid-19 no Brasil. Ciênc. saúde coletiva, 25(Suppl 2): 4201-4210.

Guimarães, F. J., Santos, F. J. S., Leite, A. F. B., Holanda, V. R., Sousa, G. S. \& Perrelli, J. G. A. (2019). Adoecimento mental em gestantes. Enfermería Global, 53: 511-522.

Lana, R. M, Coelho, F. C., Gomes, M. F. C., Cruz, O. G., Bastos, L. S., Villela, D. A. M. \& Codeço, C. T. (2020). Emergência do novo coronavírus (SARSCoV-2) e o papel de uma vigilância nacional em saúde oportuna e efetiva. Cad. Saúde Pública, 36(3): e00019620.

Lebel, C., MacKinnon, A., Bagshawe, M., Tomfohr-Madsen, L. \& Giesbrecht, G. (2020). Elevated depression and anxiety symptoms among pregnant individuals during the COVID-19 pandemic. J Affect Disord, 277: 5-13.

Lélis, B. D. B., Correa, J. M. C., Marinho, G. P., Alves, K. M., Duarte, J. V. B., Marinho, I. P. \& Barnardes, N. B. (2020). O Sofrimento Mental das Gestantes em Meio a Pandemia do Novo Coronavírus no Brasil. Id on Line Rev. Mult. Psic, 14(52): 442-451.

Lockwood, C., Porrit, K., Munn, Z., Rittenmeyer, L., Salmond, S., Bjerrum, M., Loveday, H., Carrier, J \& Stannard, D. (2017). Chapter 2: Systematic reviews of qualitative evidence. In: Aromataris E, Munn Z (Editors). Joanna Briggs Institute Reviewer's Manual. The Joanna Briggs Institute,

Mascarenhas, V. H. A., Becker, A. C., Venâncio, K. C. M. P., Baraldi, N. G., Durkin, A. C., \& Riesco, M. L. G. (2020). COVID-19 e a produção de conhecimento sobre as recomendações na gravidez: revisão de escopo. Revista Latino-Americana de Enfermagem, 28(4): e3348. 10.1590/15188345.4523 .3348

Mendes, K. D. S., Silveira, R. C. C. P. \& Galvão, C. M. (2008). Revisão integrativa: método de pesquisa para a incorporação de evidências na saúde e na enfermagem. Texto Contexto Enferm, 17(4): 758-764. 
Research, Society and Development, v. 10, n. 15, e329101522656, 2021

(CC BY 4.0) | ISSN 2525-3409 | DOI: http://dx.doi.org/10.33448/rsd-v10i15.22656

Oliveira, W. K., Duarte, E., França, G. V. A. \& Garcia, L. P. (2020). Como o Brasil pode conter o COVID-19. Epidemiol. Serv. Saúde, 29(2): e2020044.

Sade, S. Sheiner, E., Wainstock, T., Hermon, N., Yaniv Salem, S., Kosef, T., Lanxner Battat, T., Oron, S. \& Pariente, G. (2020). Risk for Depressive Symptoms among Hospitalized Women in High-Risk Pregnancy Units during the COVID-19 Pandemic. J Clin Med, 9(8): 2449

Souza, D. O. (2020). A pandemia de COVID-19 para além das Ciências da Saúde: reflexões sobre sua determinação social. Ciênc. saúde coletiva, 25(Suppl 1): 2469-2477.

Souza, A. R., Mendes, M. H., Ximenes, T.V., Moura, A. D. A., Lima, G. G. \& Feitoza, A. R. (2017). Gestação de mulheres portadoras de transtorno mental. Rev. Tendên. da Enferm. Profis, 9(1): 2089-2094.

Whittemore, R., \& Knafl, K. (2005). The integrative review: updated methodology. J Adv Nurs, 2(5): 546-53.

Zhou, Y., Shi, H., Liu, Z., Peng, S., Wang, R., Qi, L., Li, Z., Yang, Z.,Ren, Y., Song, X., Zeng, L., Qian, W. \& Zhang, X. (2020). The prevalence of psychiatric symptoms of pregnant and non-pregnant women during the COVID-19 epidemic. Translational Psychiatry, 10: 319. 